\title{
Diagnosis and Management of the Venous Malformations of Klippel-Trenaunay Syndrome
}

\author{
S. Keisin Wang MD ${ }^{1,2}$, Natalie A. Drucker MD ${ }^{1}$, Alok K. Gupta ${ }^{1,2}$, Francis E. \\ Marshalleck $\mathrm{MD}^{3}$, and Michael C. Dalsing $\mathrm{MD}^{1,2}$ \\ Indiana University School of Medicine, ${ }^{1}$ Departments of Surgery, ${ }^{2}$ Division of Vascular \\ Surgery, and ${ }^{3}$ Department of Radiology
}

\author{
Corresponding Authors: \\ Michael C. Dalsing, MD \\ mdalsing@iupui.edu \\ 1801 N. Senate St \\ Suite 3500 \\ Indianapolis, IN 46202 \\ S. Keisin Wang, MD \\ wangkei@iupui.edu \\ 1801 N. Senate St \\ Suite 3500 \\ Indianapolis, IN 46202
}

This is the author's manuscript of the article published in final edited form as:

Wang, S. K., Drucker, N. A., Gupta, A. K., Marshalleck, F. E., \& Dalsing, M. C. (2017). Diagnosis and management of the venous malformations of Klippel-Trénaunay syndrome. Journal of Vascular Surgery: Venous and Lymphatic Disorders, 5(4), 587-595. https://doi.org/10.1016/j.jvsv.2016.10.084 


\begin{abstract}
:
Objective

A dearth of information exists in the literature regarding current practice in the management of Klippel-Trenaunay Syndrome (KTS), a rare condition. We seek to review and describe the etiology, diagnosis, and treatment of the KTS patient.
\end{abstract}

\title{
Methods
}

Relevant data was synthesized from a Medline review using a combination of key terms “Klippel” and “Trenaunay”. Majority of hits described singular case reports and were subsequently excluded. Remaining papers were then reviewed and included based on quality of evidence and author discretion.

\section{Conclusion}

KTS is characterized by a clinical triad of extremity varicosities, cutaneous vascular malformations, and hypertrophy of soft tissues and/or long bones. The diagnosis is clinically supplemented with MRI and CT scanning. Although this syndrome is associated with significant comorbidities such as pain, edema, ulcerations, and pruritus it is rarely the cause of mortality. The backbone of treatment is nonoperative in nature but should be supplemented with minimally invasive, endovascular, and rarely open surgical procedures for refractory cases. 


\section{Introduction:}

Vascular malformations of Klippel-Trenaunay syndrome (KTS) usually affect the capillary, venous, and lymphatic systems of the lower extremities. Rarely, these defects are seen in the upper extremities, are bilateral, or involve the trunk. Although first described by Hilaire in 1832, KTS was truly recognized as an entity in itself by its namesakes at the turn of the $20^{\text {th }}$ century. ${ }^{(1)}$ The duo of Frenchmen defined a cohort of patients presenting with a triad of asymmetric limb hypertrophy, localized capillary malformation (nevus flammeus, “port-wine stain”), and congenital lower extremity varicosities. Although KTS is generally benign in course, the diseased limb can exhibit pain, swelling, hyperpigmentation, thrombophlebitis, variceal bleeding, and ulceration.

Shortly after description by Klippel and Trenaunay, Weber designated a similar vascular syndrome with the addition of arteriovenous fistulas (AVFs) termed the ParkesWeber Syndrome (PWS). ${ }^{(2)}$ This separate pathology has been the source of much confusion since the mid- $20^{\text {th }}$ century as clinicians have often ascribed one as the other. However, there are crucial differences which affect the clinical course. With addition of high flow AVFs, those suffering from PWS have a decreased lifespan and more significant arterial complications compared to the more benign KTS.

Much effort has been made to unify the criteria of KTS in order to clarify the clinical condition, the first step in diagnosis and management. Currently, the best classification of venous malformations, of which KTS is one, is the Hamburg 
Classification of Congenital Vascular Malformations. Utilizing this tool, KTS would be classified as a predominantly venous defect of the truncular anatomic form presenting with aplasia, obstruction, and/or dilatation. Truncular forms arises after developmental arrest and during vascular trunk formation; in other words, these lesions do not have the ability to proliferate after removal. ${ }^{(3,4)}$

The true pathogenesis of KTS has been elusive. While most patients demonstrate a normal karyotype, sporadic translocations have been reported of chromosomes 5-11 and 8-14; a supernumerary ringed chromosome 18 have also been described. Familial cases are rarely (and often unreliably) reported. ${ }^{(5)}$ The affected limbs tend to exhibit increased blood flow; this has been thought related to VEGF-mediated angiogenesis, but no definitive evidence has been brought forth. There also may be involvement of gain-offunction PIK3CA gene mutations causing upregulation of AKT and mTOR signaling. ${ }^{(6)}$ PI3K and mTOR inhibitors are currently being investigated as treatment options with promising results in mice and humans. ${ }^{(7-9)}$ At least 6 hypothetical causes of KTS have been suggested in the literature; of which, none completely explains the entire clinical spectrum. ${ }^{(5)}$ 


\section{Presentation:}

KTS is clinically diagnosed by the observation of a triad of cutaneous capillary malformations, varicosities, and hypertrophy soft tissues and/or long bones. However, a spectrum and combination of the aforementioned is usually appreciated in the real world. In addition, while this classical presentation is usually observed in a singular limb, multiple and even whole body cases have been described. ${ }^{(10)}$

Limb length discrepancy is most often secondary to underlying soft tissue growth but can be associated with long bone hypertrophy. The difference in growth occurs during childhood before stabilizing in young adulthood with minimal changes after the teenage years. ${ }^{(11)}$

Capillary malformations present on the hypertrophied limb and clinically appear as flat hemangiomas. In contrast, true flow-limiting arterial pathology is exceedingly rare in KTS and more characteristic of the more pathologic PWS. A cutaneous capillary malformation is often the first and most obvious sign on examination of the patient suspected of KTS. This is often referred to in the literature as a "port-wine stain". Frequently dermatomal in distribution, these lesions tend to bleed and persist as the patient ages. The frequency of which these skin findings coexist in KTS cannot be understated; in fact, the prevalence of capillary malformations approaches $100 \%$ in the reported literature. ${ }^{(12)}$ 
Varicosities usually appear on the lateral aspect of the limb resulting from persistent embryonic veins, superficial venous malformations, or as a result of deep venous aberrations consisting of hypoplasia, segmental aplasia, and aneurysmal degeneration. ${ }^{(2,13)}$ Varicosities are slightly less common than capillary malformations and range from $76-100 \%$ in small case series (Figure 1). ${ }^{(12)}$ These pathologic vessels disappear proximally as the diseased veins dive into the proximal femoral and iliac systems. Patients mainly complain of swelling, often with pain, but may also experience more advanced clinical signs and symptoms of chronic venous disease. ${ }^{(14,15)}$

Frequently, patients present with a persistent embryonic vein on the lateral aspect of the distal leg termed a "lateral marginal vein" or "persistent sciatic vein”. Prevalence rates in the literature range from $9-68 \%{ }^{(13,15-19)}$ Animal fetal studies have demonstrated these embryonic veins form and regress by 12-weeks of life, but this has yet to be demonstrated in humans. ${ }^{(19)}$

Yamaki et al reported the frequency of venous and lymphatic malformations in 61 KTS patients clinically diagnosed with duplex and MRI. Capillary malformations were detected in $89 \%$, among which port-wine stain was most prominent (66\%), followed by telangiectasia (51\%) and angiokeratoma (30\%). A persistent embryonic vein was detected in 53\% of the patients. Reflux was observed in the GSV (20\%), embryonic vein (15\%), and SSV (7\%). Deep vein hypoplasia/aplasia was observed in 20\%.

Extratruncular and truncular lymphatic malformations were observed in $21 \%$ and $28 \%$ of the patients respectively. ${ }^{(20)}$ 
The signs and symptoms of KTS are often related to the obstruction or insufficiency present in the venous outflow and can be scored using the CEAP classification system for chronic venous disease (clinical presentation, etiology, anatomic involvement, and pathophysiology). ${ }^{(3,21)}$ Furthermore, the results of any treatment should be determined using Venous Severity Scores and Quality of Life questionnaires used for more familiar forms of chronic venous disorders. ${ }^{(3,15,22)}$

If internal organs are affects by vascular malformations, patients may report mild hematuria or hematochezia. ${ }^{(23)}$ In a small, and unfortunate subpopulation, bleeding may be intractable and necessitate bowel resection. ${ }^{(10)}$ 


\section{Diagnosis:}

The diagnosis of KTS is clinical and difficult to make even for the experienced physician as there is no true pathognomonic test. However, an elevated D-dimer and mutation of the AGGF1 gene may suggest the diagnosis. ${ }^{(4)}$ The status of the PIK3CA gene does not currently add diagnostic value but may yield useful information in the

future. ${ }^{(24)}$ Duplex ultrasonography, Magnetic Resonance (MR) imaging, and Computerized Tomographic (CT) imaging may show soft tissue hypertrophy and associated vascular malformations. Plain films can be used to screen long bones for limb length discrepancies.

Patients suspected of KTS should be initially evaluated with noninvasive imaging. The purpose of which is to determine the extent and severity of disease along with delineation of any significant arteriovenous shunting which may be present. ${ }^{(21)}$

The high sensitivity and specificity of lower extremity duplex imaging in regards to venous and arterial anatomy and function makes it the best initial diagnostic study. ${ }^{(16)}$ Confirmation can be easily obtained of valvular incompetence, low-flow regions, compressible vascular channels, and anatomical abnormalities such as aneurysms or atretic segments quickly, at low cost, and without the need for sedation in the pediatric population. In addition to B-mode and color flow, hemodynamic studies such as ankle/brachial indices, segmental pressures, and pulse volume recordings may be useful 
in flushing out suspected arterial anomalies such as the blunted waveforms and reduced digital pressures observed distal to a high flow AVM.

Most consider MR imaging essential in the diagnostic evaluation of patients with KTS as it allows differentiation of the soft tissues. This modality can provide the first assessment of the involvement of muscles, joints, and bones. Also, the addition of IV gadolinium contrast can discriminate venous from the more insidious arterial malformations and help the classification of patients into the Hamburg system. ${ }^{(21)}$

CT imaging incurs a radiation dose along with potential renal toxicity depending on patient's prestudy renal function and contrast usage. It may not provide detail of muscle and surrounding soft tissue involvement, for which the MR excels. Additionally, many patients undergoing initial evaluation for KTS are children, making the avoidance of radiation all the more important. However, contrast CT imaging with venous phase protocols may be of particular use in preinterventional planning and cannot be avoided at times despite the best intentions.

Lastly, ascending venography should be employed to assist with preoperative planning when superficial vein eliminating procedures such as ablation, sclerotherapy, ligation, or stripping are planned as it can be performed concurrently. If documentation of a patent deep venous system has not been performed previously, venography can assist with this as well. 
The differential diagnosis for patients being considered for KTS should include Sturge-Weber syndrome, Parkes-Weber syndrome, lymphatic filariasis, BeckwithWiedemann syndrome, Russell-Silver syndrome, CHILD syndrome, NF1, triploid syndrome, and Proteus syndrome. ${ }^{(24)}$ The differences in presentation of these complex disease states are briefly delineated in table I. The plethora of named syndromes makes the Hamburg classification so appealing in that it provides a structure and delivers direction to treatment. 


\section{Medical Management:}

Non-operative medical management is the main modality in the treatment of symptomatic KTS patients. Rarely, and only in patients refractory to medical management, should operative intervention be considered. For example, in a series of 19 KTS patients reported by Sung et al, only 4 underwent operative intervention during a mean follow-up of 4.1 years with no major adverse outcomes regardless of management style. $^{(14)}$

Patients with KTS should be managed similarly to those with more common varieties of chronic venous disorders with appropriate consideration of potentially extenuating circumstances such as deep venous agenesis (Figure 2). The latter may make the remaining superficial veins a very important egress for lower extremity outflow.

Patients presenting with symptoms of chronic venous disease should be initiated on a regimen of compression and elevation. This is the fundamental basis of all treatment related to limb edema. Compression stockings should extend from above the affected area to the digits and should be fitted to the individual limb. The correct pressure may vary for the patient but typically ranges from 20-40 mmh. However, compliance to this garment can be difficult in the pediatric population with rapidly growing limbs along with other social factors. Low-dose aspirin should be considered as there is limited benefit to pain and swelling in patients with vascular malformations. ${ }^{(25)}$ If symptoms are refractory to compression alone, intermittent pneumatic compression can be an useful 
adjunct in lower extremity edema mobilization. ${ }^{(26)}$ Some clinicians will trial a course of diuretics in the patient with continued symptomatic edema despite compression, mobilization, and elevation; however, there is no evidence of efficacy in reduction of limb edema. ${ }^{(17)}$

If soft tissue inflammation develops, it should initially be managed with over the counter analgesics and elevation. Be mindful this presentation is often initially sterile; therefore, antibiotics should be reserved until cellulitis with virulent organisms is suspected. ${ }^{(17)}$ Wound cultures can be performed, however, organisms are rarely isolated. There is currently no evidence for prophylactic antibiotics in this population.

Additionally, the need for careful wound care and personal hygiene should be reinforced with each clinic visit.

No role for anticoagulation has been identified early in the clinical course; however, it should be initiated if patients develop deep venous thrombosis (DVT). Additionally, adults who have had a venous intervention should be considered for a short perioperative course of prophylaxis, as they have increased risk for thromboembolic events. ${ }^{(11)}$ Additionally, women with severe venous malformations should avoid oral contraceptives as this has been associated with a higher risk of thromboembolic events. $^{(17)}$

Limb length discrepancies can be appropriately managed with heel inserts or compensatory shoes to avoid scoliosis as long as discrepancies are less than $1.5 \mathrm{~cm}$. 
However, if the discrepancy in limb length is greater than $2 \mathrm{~cm}$, surgical intervention should be considered in the form of osteotomy or epiphysiodesis by the appropriately trained orthopedic surgeon.

Using the CEAP classification, operative vascular intervention should be considered when the severity of disease progresses to greater than class 3 or potentially class 2 in some symptomatic cases. ${ }^{(27)}$ The largest experience of vascular malformations intervened upon are reported by Mayo Clinic. In their practice, absolute indications for intervention consists of continuing hemorrhage, refractory ulcerations, and acute thromboembolism. Relative indications consists of pain, functional impairment, swelling secondary to venous insufficiency, limb asymmetry, and cosmesis. ${ }^{(21)}$

Because of the rarity and complexity of KTS, early referral to specialist centers should be considered. ${ }^{(24)}$ The varying presentations and severity of disease necessitates that each treatment plan be individualized for the specific KTS patient. Additionally, a multidisciplinary team should be involved with the patient's care so that all needs are addressed as they manifest. Perhaps the most important member of the team is the patient. He/she should be educated regarding the nature of their disease as compliance to any proposed treatment is essential to ultimate success and only increases with education. 


\section{Endovascular and Minimally Invasive Intervention:}

The role of endovascular therapy and minimally invasive intervention in KTS is not well-established secondary to the low prevalence of the disease; however, it should be offered over open surgery after failed nonoperative management. The basis of the success of sclerotherapy, embolization, ablation, and laser treatments in KTS are built from the parallel experience in chronic venous and capillary disease.

A plastic surgery group from South Korea described pulsed dye laser therapy in their KTS population for capillary malformations, namely port-wine stains. 4 of their small series of 19 patients were treated with laser without morbidity. The 585nm wavelength has also been effectively used in the treatment of facial telangiectasias and hemangiomas. ${ }^{(14)}$ The clinician should keep in mind lighter colored lesions in the pediatric population respond best while darker lesions in adults are more refractory to treatment; however, these darker lesions are responsive to nontraditional Krypton and copper lasers. ${ }^{(28)}$

After failed non-operative management of varicosities, sclerotherapy or embolization (Figure 3) techniques with various agents can be attempted. However, before intervention is performed, the deep venous system should be documented to be patent by imaging of choice. These techniques, over RFA ablation, should be considered for persistent embryonic and large superficial veins to avoid post-operative persistence of hard cords and thermal injury. ${ }^{(24)}$ In particular, the persistent sciatic vein should be 
treated with sclerotherapy or embolization secondary to close proximity of the sciatic nerve. ${ }^{(24,29)}$

Burrows et al reported their series of consecutive sclerotherapy patients with low flow vascular malformations and noted a good or excellent result in up to $90 \%$ of patients by clinical survey. However, there was a complication rate of $12 \%$ per treatment and a $28 \%$ complication rate per patient. Almost $15 \%$ of the patients in this series had some evidence of skin necrosis. To decrease the high-risk of complications, the authors cautioned against injection of agents, especially ethanol, close to major nerves or cutaneous lesions. ${ }^{(30)}$ Later reports have echoed these initial findings. ${ }^{(31)}$

Foam sclerotherapy has also been well documented in the literature. Smith reports a series of 808 mostly CEAP class 2 patients undergoing ultrasound-guided injection with diluted sodium tetradecyl. Although follow-up was marginal, there was a significant decrease in CEAP classification 6 months after the procedure. Minor complications were minimal, and the author reported no major complications. ${ }^{(32)}$

Endovascular radiofrequency ablation of the persistent embryonic or GSV has also increasingly gained popularity over the last 10 years. Weiss et al reports 934 consecutive radiofrequency and laser thermal ablations of superficial veins with 6-month, 1-year, and 5-year ablation rates at 92\%, 86\%, and 72\% respectively. ${ }^{(33)}$ No differences were noted in the laser and radiofrequency ablation groups. Smaller series on KTS patients also mirror these excellent results, although retreatment is common, with 
minimal complications. ${ }^{(34)}$ As with superficial veins in any patient, care has to be taken when using these modalities and no heat sink can be provided during the procedure. The result could be significant cutaneous burns. ${ }^{(35)}$ 


\section{Open Surgery:}

Although some advocate for early surgical intervention, ${ }^{(36)}$ open surgical volume has drastically decreased over the last several decades and become more of historical interest secondary to wound complications and persistent bleeding. Currently, open operations are reserved for patients who are not candidates for minimally invasive techniques and, therefore, mainly consist of a combination of high ligation of persistent embryonic veins, vein stripping, and stab phlebectomies. Subfascial endoscopic perforator surgery (SEPS) has been useful in the select group of patients with large and incompetent perforating veins but new available percutaneous interventions are making this of historical significance as well.

Ulcerated or bleeding lesions may be excised when possible; however, the clinician should keep in mind the poor wound healing potential of the KTS limb. ${ }^{(37)}$ Therefore, post-operative edema should be kept to a minimum with compression and elevation as tolerated. Before intervention, the venous anatomy should be meticulously documented to avoid destroying the superficial venous system in patients with an atretic deep system.

Because of the robust venous network that can develop in these patients, consideration should be given to the potential blood loss during an open operation. Blood should be available in the operating theatre to transfuse as needed. Additionally, tourniquets can be an important adjunctive tool and should be used when appropriate. ${ }^{(12)}$ 
The largest open surgical series comes from the French surgeon Servelle who described 786 "KTS" operations over 40 years of practice in 1985. Although the author claimed all patients were clinically diagnosed with KTS, $71 \%$ of the operations were performed for venous compression release in the extremities, not a problem often seen in the KTS population. Only $36 \%$ of the cohort had clinically apparent varicosities while port-wine stains were only present in $32 \%$. Follow-up and outcomes were not reported. ${ }^{(18)}$

Baraldini et al reported their experience of 29 pediatric KTS patients undergoing a combination of open and minimally invasive interventions for venous disease. Average age of intervention was 10.3 years. The procedures were all technically successful. No mortalities or major complications were noted on 6-month and 2-year follow-up. The authors suggest early intervention is safe and efficacious in minimizing long-term venous hypertension and its sequelae. ${ }^{(36)}$

The Mayo Clinic group documented their surgical experience longitudinally in consecutive KTS patients over two decades previous to the widespread adoption of RFA and laser ablation. One such report describes intervention in 53 limbs of 49 patients. The average length of follow up was 73 months. All had varicosities, 73\% had limb hypertrophy, and all patients met two of the three diagnostic KTS criteria. The most common presenting symptom was disabling pain (88\%). Stripping of the GSV, small SV, and lateral embryonic vein was undertaken in 32\%, 36\%, and 28\% respectively. 
Short-term morbidity included a 4\% DVT risk, a 14\% thrombophlebitis rate, and 10\% experienced at least one episode of cellulitis. There were no deaths. 13/49 patients required reintervention. Nonetheless, 50\% reported significant pain relief and statistically significant decreases in VCSS and CEAP scores were reported. ${ }^{(12)}$ 


\section{Conclusion:}

Klippel-Trenaunay Syndrome is characterized by a triad of cutaneous vascular malformations in the form of port-wine stains, limb length discrepancies, and venous insufficiency secondary to venous malformations.

The diagnosis of this disease is clinical but can be supplemented with noninvasive imaging such as duplex sonography to define the lower extremity venous anatomy. Radiological studies such as MR (primarily) and CT imaging can further delineate the deep and superficial venous anatomy as well as adjacent soft tissue, joint, muscle and bony involvement so needed for preoperative planning.

Primary treatment consists of nonoperative management with lifestyle modification, local wound care, orthotics, extremity elevation, and compression therapy. Operative intervention is rarely required. If symptom progression occurs, patients should be offered a combination of pulsed laser therapy, embolization, sclerotherapy with foam or ethanol, and/or radiofrequency or laser ablation.

Patients who are not candidates for minimally invasive techniques may benefit from open surgery consisting of vein stripping, stab phlebectomies, or more aggressive venous reconstructions. It is important to image the deep system to confirm patency prior to removal of flow from the superficial system. 


\section{References:}

1. Klippel M, Trenaunay P. Du Naevus Variqueux Osteo-hypertrophique. Memoires Originaux. 1900.

2. Baskerville PA, Ackroyd JS, Lea Thomas M, Browse NL. The KlippelTrenaunay syndrome: clinical, radiological and haemodynamic features and management. Br J Surg. 1985.

3. Marshalleck F DM. Venous Malformations: Pathophysiology, Classification, and Incidence. In: Davies MG LA, editor. Chronic Venous Insufficiency:

Contemporary Endovascular Management. 12011. p. 189-97.

4. Lee BB, Laredo J, Lee TS, Huh S, Neville R. Terminology and classification of congenital vascular malformations. Phlebology. 2007.

5. Oduber CE, van der Horst CM, Hennekam RC. Klippel-Trenaunay syndrome: diagnostic criteria and hypothesis on etiology. Ann Plast Surg. 2008.

6. Luks VL, Kamitaki N, Vivero MP, Uller W, Rab R, Bovee JV, et al. Lymphatic and other vascular malformative/overgrowth disorders are caused by somatic mutations in PIK3CA. J Pediatr. 2015.

7. Castel P, Carmona FJ, Grego-Bessa J, Berger MF, Viale A, Anderson KV, et al. Somatic PIK3CA mutations as a driver of sporadic venous malformations. Science Translational Medicine. 2016.

8. Castillo SD, Tzouanacou E, Zaw-Thin M, Berenjeno IM, Parker VER, Chivite I, et al. Somatic activating mutations in Pik3ca cause sporadic venous malformations in mice and humans. Science Translational Medicine. 2016.

9. Adams DM, Trenor CC, Hammill AM, Vinks AA, Patel MN, Chaudry G, et al. Efficacy and Safety of Sirolimus in the Treatment of Complicated Vascular Anomalies. Pediatrics. 2016.

10. Sreekar H, Dawre S, Petkar KS, Shetty RB, Lamba S, Naik S, et al. Diverse manifestations and management options in Klippel-Trenaunay syndrome: A single centre 10-year experience. Journal of Plastic Surgery and Hand Surgery. 2013.

11. Kihiczak GG, Meine JG, Schwartz RA, Janniger CK. Klippel-Trenaunay syndrome: a multisystem disorder possibly resulting from a pathogenic gene for vascular and tissue overgrowth. Int J Dermatol. 2006.

12. Malgor RD, Gloviczki P, Fahrni J, Kalra M, Duncan AA, Oderich GS, et al. Surgical treatment of varicose veins and venous malformations in KlippelTrenaunay syndrome. Phlebology. 2016.

13. Baskerville PA, Ackroyd JS, Browse NL. The etiology of the Klippel-Trenaunay syndrome. Ann Surg. 1985.

14. Sung HM, Chung HY, Lee SJ, Lee JM, Huh S, Lee JW, et al. Clinical Experience of the Klippel-Trenaunay Syndrome. Arch Plast Surg. 2015.

15. Delis KT, Gloviczki P, Wennberg PW, Rooke TW, Driscoll DJ. Hemodynamic impairment, venous segmental disease, and clinical severity scoring in limbs with Klippel-Trenaunay syndrome. J Vasc Surg. 2007.

16. Howlett DC, Roebuck DJ, Frazer CK, Ayers B. The use of ultrasound in the venous assessment of lower limb Klippel-Trenaunay syndrome. European Journal of Radiology. 1994. 
17. Jacob AG, Driscoll DJ, Shaughnessy WJ, Stanson AW, Clay RP, Gloviczki P. Klippel-Trenaunay syndrome: spectrum and management. Mayo Clin Proc. 1998. 18. Servelle M. Klippel and Trenaunay's syndrome. 768 operated cases. Ann Surg. 1985.

19. Oduber CE, Young-Afat DA, van der Wal AC, van Steensel MA, Hennekam RC, van der Horst CM. The persistent embryonic vein in Klippel-Trenaunay syndrome. Vasc Med. 2013.

20. Yamaki T, Konoeda H, Fujisawa D, Ogino K, Osada A, Hamahata A, et al. Prevalence of various congenital vascular malformations in patients with KlippelTrenaunay syndrome. Journal of Vascular Surgery: Venous and Lymphatic Disorders. 2013.

21. Gloviczki P, Driscoll DJ. Klippel-Trenaunay syndrome: current management. Phlebology. 2007.

22. Vasquez MA, Rabe E, McLafferty RB, Shortell CK, Marston WA, Gillespie D, et al. Revision of the venous clinical severity score: venous outcomes consensus statement: special communication of the American Venous Forum Ad Hoc Outcomes Working Group. J Vasc Surg. 2010.

23. Li T, Hu S-Y, Chen Z-T, Chen Z-Q, Zhi X-T. Colorectal cavernous hemangioma in Klippel-Trenaunay syndrome: A rare cause of abdominal pain and hematochezia. Surgery. 2015.

24. Group KW. Clinical Practice Guidelines for Klippel-Trenaunay Syndrome. Boston Children's Hospital [Internet]. 2016. Available from: https://kt.org/assets/images/content/BCH-Klippel-Trenaunay-Syndrome-ManagementGuidelines-1-6-2016.pdf.

25. Nguyen JT, Koerper MA, Hess CP, Dowd CF, Hoffman WY, Dickman M, et al. Aspirin Therapy in Venous Malformation: A Retrospective Cohort Study of Benefits, Side Effects, and Patient Experiences. Pediatric Dermatology. 2014.

26. Capraro PA, Fisher J, Hammond DC, Grossman JA. Klippel-Trenaunay syndrome. Plast Reconstr Surg. 2002.

27. Eklof B, Rutherford RB, Bergan JJ, Carpentier PH, Gloviczki P, Kistner RL, et al. Revision of the CEAP classification for chronic venous disorders: consensus statement. J Vasc Surg. 2004.

28. Han G. Applications of lasers in medical dermatology. Cutis. 2014.

29. Mattassi R, Vaghi M. Management of the marginal vein: current issues. Phlebology. 2007.

30. Burrows PE, Mason KP. Percutaneous treatment of low flow vascular malformations. J Vasc Interv Radiol. 2004.

31. Lee BB, Do YS, Byun HS, Choo IW, Kim DI, Huh SH. Advanced management of venous malformation with ethanol sclerotherapy: mid-term results. J Vasc Surg. 2003.

32. Smith PC. Chronic venous disease treated by ultrasound guided foam sclerotherapy. Eur J Vasc Endovasc Surg. 2006.

33. Weiss RA, Weiss MA, Eimpunth S, Wheeler S, Udompunturak S, Beasley KL. Comparative outcomes of different endovenous thermal ablation systems on great and small saphenous vein insufficiency: Long-term results. Lasers Surg Med. 2015. 
34. Frasier K, Giangola G, Rosen R, Ginat DT. Endovascular radiofrequency ablation: a novel treatment of venous insufficiency in Klippel-Trenaunay patients. J Vasc Surg. 2008.

35. Gale SS, Lee JN, Walsh ME, Wojnarowski DL, Comerota AJ. A randomized, controlled trial of endovenous thermal ablation using the 810-nm wavelength laser and the ClosurePLUS radiofrequency ablation methods for superficial venous insufficiency of the great saphenous vein. J Vasc Surg. 2010.

36. Baraldini V, Coletti M, Cipolat L, Santuari D, Vercellio G. Early surgical management of Klippel-Trenaunay syndrome in childhood can prevent long-term haemodynamic effects of distal venous hypertension. J Pediatr Surg. 2002.

37. Gates PE, Drvaric DM, Kruger L. Wound healing in orthopaedic procedures for Klippel-Trenaunay syndrome. J Pediatr Orthop. 1996. 


\section{Legends}

Figure 1. Patient with unilateral limb swelling secondary to persistent lateral marginal vein (yellow arrow) and varicosities (red arrow) since birth. A large and diffuse portwine stain is noted over the posterior aspect of the right thigh (green arrow).

Figure 2A. Representative axial cut of a lower extremity MRV in which an absent deep vein is noted in the left lower extremity (smaller pink arrow). A large persistent and patent lateral marginal vein appears to drain most of the left lower extremity. Normal venous anatomy is noted in the right lower extremity and highlights the normal popliteal vein (stout smaller yellow arrow).

Figure 2B. Coronal section of the patient in 2A demonstrating a normal deep vein on the right side (yellow arrow). On the left side, the deep veins are atretic (red arrow) and there is a large lateral subcutaneous vein (blue arrow) draining the left lower extremity.

Figure 3A. Axial sections from an MRV demonstrating a large left lower extremity persistent lateral marginal vein (large blue arrow) in addition to a normal patent popliteal vein (small red arrow). The opposite side is normal with the popliteal vein highlighted by a yellow arrow.

Figure 3B. Representative intraoperative imaging of the same patient as Figure 3A in which the lateral marginal vein was cannulated and subsequently embolized using metal coils.

The first panel is an image of the large lateral vein (green arrow) with only a perforator vein (blue) connecting it to the popliteal vein (yellow). The second panel demonstrates occlusive coils lying within the abnormal lateral vein.

Table I. Differential diagnosis and the presentation of the various syndromes and diseases in the patient suspected of KTS 